\title{
Application Specific Optimal Codec in Cognitive Environment
}

\author{
R. D. Raut \\ Assistant Professor \\ Electronics \& Comm.Deptt. \\ SRKNEC, Nagpur
}

\author{
Dr. K.D. Kulat \\ Professor \\ Electronics \& Computer Sci., Deptt \\ VNIT, Nagpur
}

\begin{abstract}
Optimization process needs a case study, including simulation to observe real time results. With the evolution of coding techniques like turbo, LDPC(Low Density Parity Check Code, Convolutional LDPC, RS(Reed Solomon) codes, etc., to choose a specific technique $\&$ its specific configuration to optimize the system performance is a critical task. Moreover FPGA implementation of these coders makes the design flexible and software defined. Heading towards the Cognitive Technology, wherein Bandwidth (BW) allocation is dynamic one has to configure the entire transceiver system to work over the available BW. Present paper is a summary of the $\mathrm{Ph}$. D. work carried out to optimize the BER(Bit error rate) performance of codec in the cognitive environment. In particular Application Specific Codec design is putt forth for Mobile \& WiMax, followed by improvement in the BER performance at higher BW. Few sample Results at every stage of simulation are also depicted.
\end{abstract}

\section{Keywords}

Turbo, LDPC, RS, FPGA, BER, Performance optimization, Cognitive

\section{INTRODUCTION}

Coding represents the fulfillment of quest, which lasted nearly 100 years, for a practical means of attaining the Shannon capacity bounds for a communication channel [1], specified by

$\mathrm{C}=\mathrm{B} \log (1+\mathrm{S} / \mathrm{N})$

When it was expected that the discovery of the ultimate codes would make any further work unnecessary, but infact the reverse seems to be the case: it has resulted in a renaissance of coding research, both in universities and in industry world-wide. This has already led to the discovery of a range of related codes of equivalent power, which are being worked on for application specific performance optimization.

Selection of a particular coding scheme for performance improvement becomes a critical task. Moreover while there is list of coding technique there is also a list of performance parameters [2]. The error coding technique for an application should be picked based on:

- Bit -Error Rate- The probability of Bit error. Measured in bits/s. We want to keep this small, typically less than $10^{-4}$.
Energy Per Bit- It is the amount of Energy contained in one information bit. It is related to transmitter power \& Bit Rate (bits/s) . Higher the energy Per Bit more immune the system becomes to channel impairments.

Coding Gain- The difference (in $\mathrm{db}$ ) in the required signalto-noise ratio to maintain reliable communications after coding is employed.

Code Rate- Consider an encoder that takes $\mathrm{k}$ information bits \& adds $r$ redundant bits, for a total of $n=k+r$ bits per codeword. So the code rate is the fraction $\mathrm{k} / \mathrm{n}$.

Latency- It can be defined as the time elapsed between a message is coded, transmitted \& decoded

\section{Design Complexity \& Implementation Cost}

\section{Channel parameters -}

Random Errors

Burst Errors

Bandwidth

Type of Channel AWGN/Raleigh Fading

To add to this there are contradicting ends

\section{Minimum distance vs. Code rate.}

In linear Block Codes adding larger number of parity bits can be instrumental in higher value of $\mathrm{d}_{\text {min }}$, thus increasing its error detection \& correction capability. However this will hamper the code rate, making the coding system inefficient.

\section{Latency vs. Error Performance}

Higher the code rate better the latency as less encoding and decoding time is required. But error performance of such a code will be poor.

\section{Complexity vs. Implementation}

Complexity often demands flexible design (software implementation) rather than a dedicated system (hardware implementation). The "hardware vs. software" contest is an old one, with the resolution lying somewhere in the middle (programmable, FPGA- based implementations) 


\section{Performance vs. Applications}

Error performance is often sacrificed to faster encoding decoding for real time applications like internet traffic.

\section{Mathematics vs. Reality}

Equations try to predict performance near to actual, they are still not the imitation of real performance.

Above all over last couple of years spectrum demand has increased many folds. Spectrum allocation policy has faced spectrum scarcity in particular spectrum bands. In contrast, a large portion of the assigned spectrum is used sporadically, leading to underutilization of significant amount of spectrum [3],[4] . Hence, dynamic spectrum access techniques were proposed to solve these spectrum inefficiency problems.

With vast literature survey carried out it was decided to work for BER performance improvement. The application chosen were Mobile Communication Systems \& WiMax. At the final stage the algorithms developed were modified using M-Flie programming, to adapt to the available BW.

To follow are Optimal Codec design for Mobile Communication System, the Novel Approach for Codec Design of WiMax System and the Spectrum Sensing Codec in the Cognitive Environment. Finally to conclude the results are summarized and future scope in the area is putt forth.

\section{OPTIMAL CODEC DESIGN FOR MOBILE COMMUNICATION SYSTEM}

The traditional codes cannot be used for mobile communication. The major difficulty of traditional codes is that, in an effort to approach the theoretical limit for Shannon's channel capacity, there is need to increase the code-word length of a linear block code or a constraint length of a convolutional code, which, in turn, causes the computational complexity of a decoder to increase exponentially. Random codes are known to achieve Shannon limit performance as $\mathrm{k}$ gets large, but at the price of a prohibitively complex decoding algorithm. Ultimately, a point is reached where complexity of decoder is so high that it becomes physically unrealizable. Also wireless channels are mostly affected by burst errors. But the existing codes are effectively suited for correcting random errors, but not burst errors. So the goal is to design a channel coding scheme for wireless communication that can operate at low signal to noise ratios and perform reliably. The way to combat the problem is to use concatenated coding, where two (or more) constituent codes are used in serial or in parallel. Concatenated coding schemes are proposed as a method for achieving large coding gains by combining two or more relatively simple building block or component codes. The Mobile Communication System Modeled [5] with Optimal Codec is as shown below in fig. 1. The performance in BER with decoder iterations is depicted in section 5 .

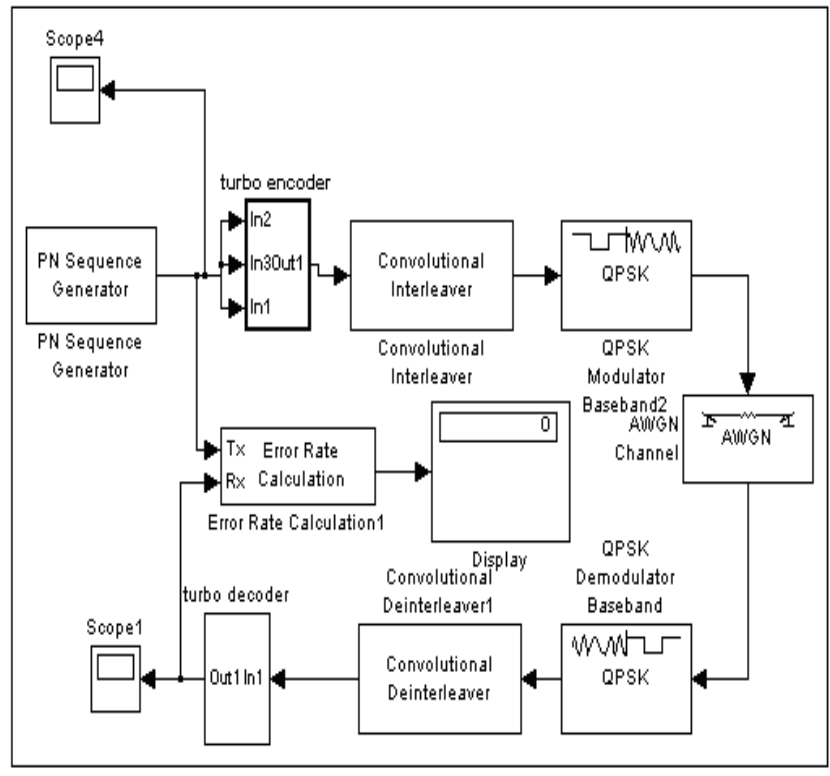

Figure 1. Simulink Model for Mobile Communication System.

\section{NOVEL APPROACH FOR CODEC DESIGN OF WIMAX SYSTEM}

For modeling the WiMax system the following major blocks were designed and implemented [6]:

\section{Data Source}

In the simulation model (fig. 3) instead of performing a randomization process, an integer source that produces random sequences of integers is used. The number of integers that are generated is specified to be frame-based and is calculated from the packet size required in each situation.

\section{RS Encoder}

As specified in the standard, the Reed-Solomon encoding is derived from a systematic RS $(\mathrm{n}=255, \mathrm{k}=239, \mathrm{t}=8)$ code using a Galois field specified as $\operatorname{GF}\left(2^{8}\right)$. The primitive and generator polynomials used for the systematic code are expressed as follows:

Primitive Polynomial:

$p(x)=x^{8}+x^{4}+x^{3}+x^{2}+1$

Generator Polynomial:

$$
g(x)=\left(x+\beta^{0}\right)\left(x+\beta^{l}\right)\left(x+\beta^{2}\right) \ldots\left(x+\beta^{2 t-1}\right)
$$

The primitive polynomial is the one used to construct the symbol field and it can also be named as field generator polynomial. The code generator polynomial is used to calculate parity symbols and has the form specified as before, where $\beta$ is the primitive element of the Galois field over which the input message is defined. To make the RS code flexible, i.e. to allow for variable block sizes and variable correction capabilities, it is shortened and punctured. When a block is shortened to $\mathrm{k}$ bytes, $239-\mathrm{k}$ zero bytes are added as a prefix, and, after the encoding process, the $239-\mathrm{k}$ 
encoded zero bytes are discarded. Once the process of shortening has been done, the number of symbols going in and out of the RS encoder change, and also the number of symbols that can be corrected $(\mathrm{t})$. With the puncturing, only the first $2 \mathrm{t}$ of the total 16 parity bytes shall be employed. Figure 2 shows the RS encoding, shortening, and puncturing process.

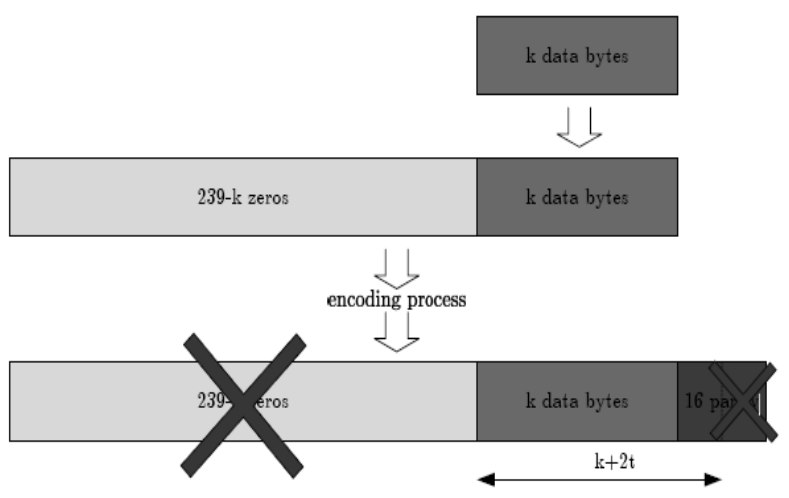

Figure 2. Process of shortening and puncturing of RS code.

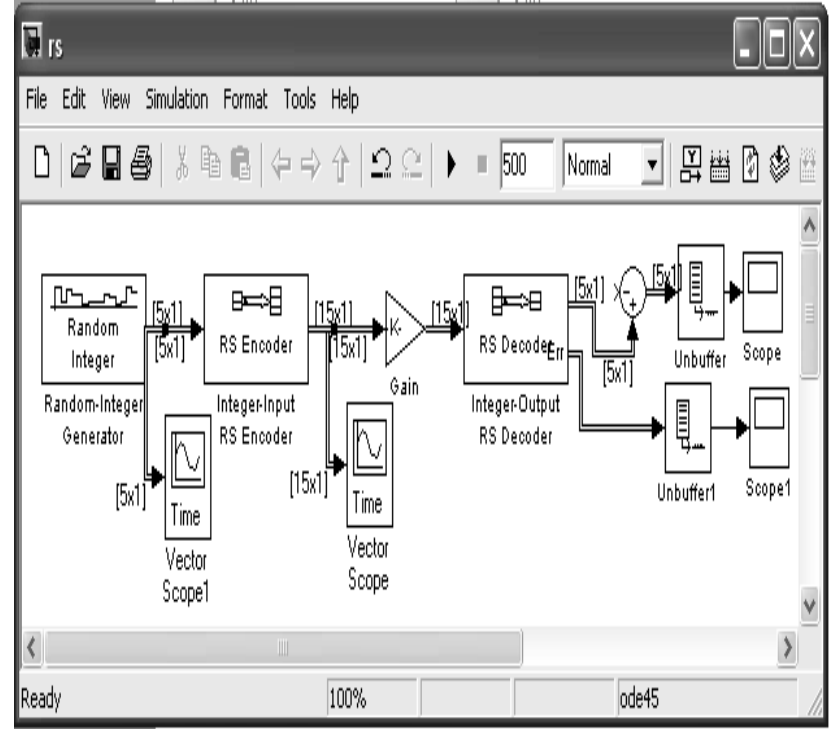

Figure 3. MATLAB Simulation of RS Encoder- Decoder for WiMax.

Convolution Encoder

IEEE WiMax 802.16d standard yields a complex convolutional encoder with a constraint length of 7 (see Figure 4).

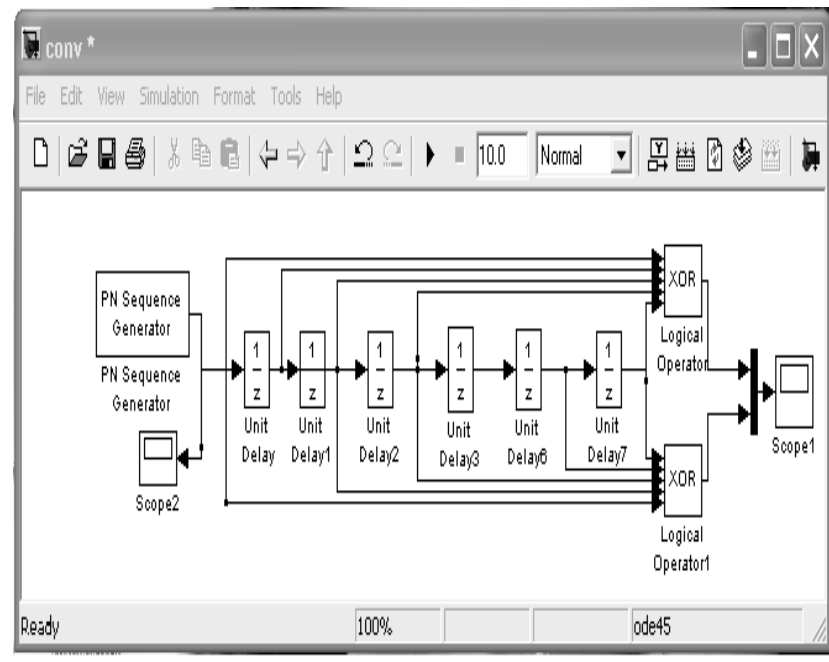

Figure 4. Matlab Simulation of Convolution Encoder.

The generator polynomials for this encoder are $\mathrm{g} 0=171$ oct and $\mathrm{g} 1=133$ oct.

The encoder can easily be implemented in hardware shift registers.

The first step is to represent the input bit string as a polynomial. Any sequence of 0 's and 1's can be represented as a binary number or a polynomial.

The convolutional encoder for WiMax ( $\mathrm{g} 0=171$ oct and $\mathrm{g} 1=$ 133 oct) can be represented as follows:

- $g 0=1+D+D^{2}+D^{3}+D^{6}$

- $g 1=1+D^{2}+D^{3}+D^{5}+D^{6}$

The convolutional encoder basically multiplies the generator polynomials by the input bit string, as follows:

- $A(x)=g 0(x) * I(x)=a b c \ldots g$

- $B(x)=g l(x) * I(x)=P Q R \ldots V$

Interleaving the two outputs from the convolutional encoder yields $E(x)=a P b Q c R \ldots g V$, which can also be written as: $x * B(x 2)$

$E(x)=\left(\begin{array}{llllll}a 0 & b 0 & c 0 & \ldots & g 0\end{array}\right)+(0 P 0 Q 0 R \ldots O V)=A(x 2)+$

Therefore, $E(x)=A(x 2)+x * B(x 2)$ and $A(x 2)=g 0(x 2) *$ $I(x 2)$ and $B(x 2)=g l(x 2) * I(x 2)$, with the following:

$$
\begin{aligned}
& \begin{aligned}
E(x) & =g 0(x 2) * I(x 2)+x * g l(x 2) * I(x 2) \\
& =I(x 2) *(g 0(x 2)+x * g l(x 2)) \\
& =I(x 2) * G(x) \text { where }
\end{aligned} \\
& G(x)=g 0(x 2)+x * g l(x 2) \\
& \text { i.e. } G(x)=1+x+x^{2}+x^{4}+x^{5}+x^{6}+x^{7}+x^{11}+x^{12}+x^{13} .
\end{aligned}
$$


It consists of two convolutional encoders. The outputs of the turbo encoder are the information sequence, together with the corresponding parity sequence produced by first encoder and the parity sequence produced by the second encoder block, the input to second encoder is through interleaver, which scrambles the data bit sequence. Simulation model of Turbo encoder -decoder is shown in fig 5 .
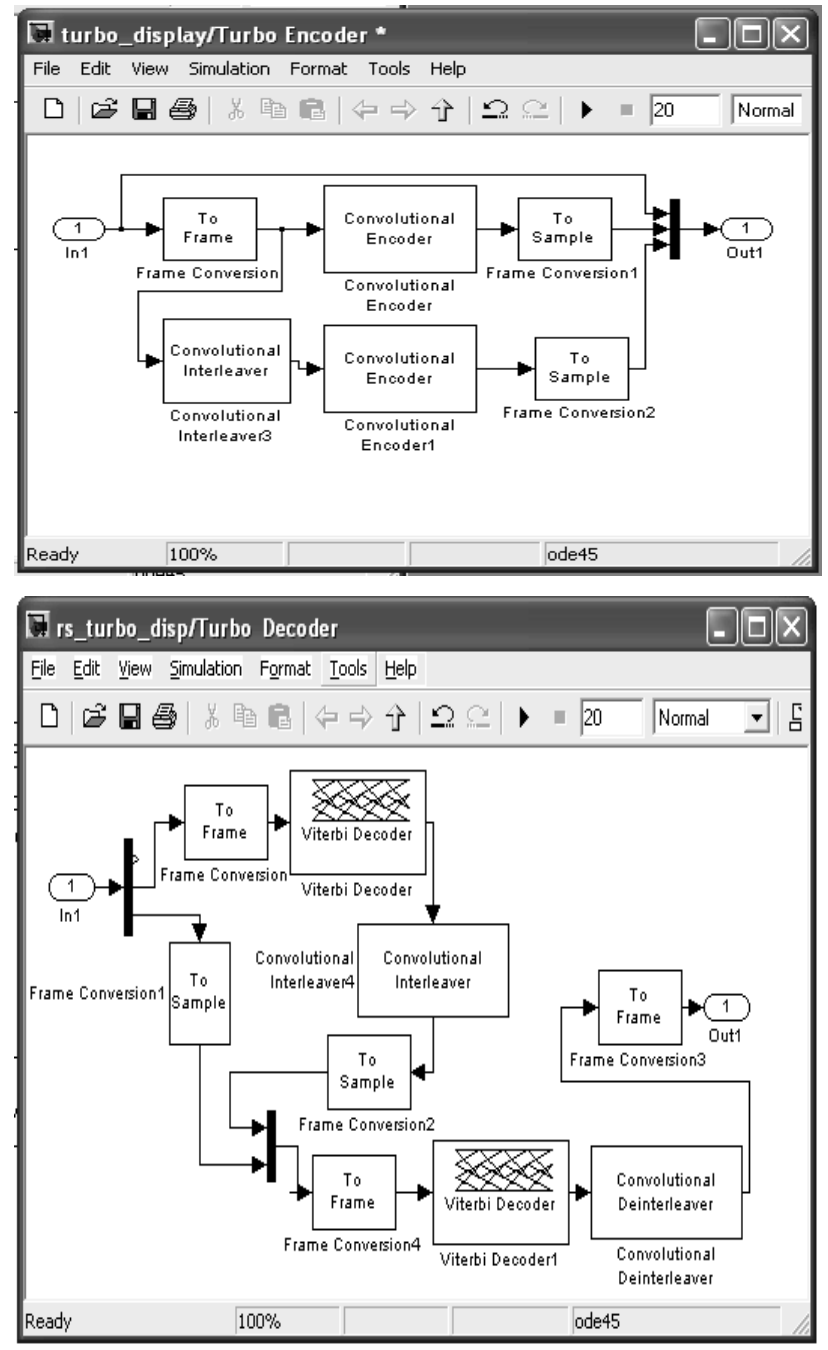

Figure 5. Simulink Model of Turbo Encoder- Decoder.

\section{Turbo Decoder}

Turbo decoder shown above in Fig 5 uses iterative decoding. The turbo code decoder is based on a modified Viterbi algorithm that incorporates reliability values to improve decoding performance. The turbo decoder consists of $\mathrm{M}$ elementary decoders - one for each encoder in turbo encoding part. Each elementary decoder uses the Soft Decision Viterbi Decoding to produce a soft decision for each received bit. After an iteration of the decoding process, every elementary decoder shares its soft decision output with the other M - 1elementary decoders.

\section{Channel}

The AWGN Channel block adds white Gaussian noise to a real or complex input signal.

Each of the major blocks mentioned above have individual sub blocks which are configured to meet the WiMax Specifications (After scaling, keeping in mind the mathematical constraints of modeling a real time system). The comparative simulations results are depicted in section5.

\section{BER IMPROVEMENT IN COGNITIVE ENVIORNMENT}

The doctoral research work is for the Spectrum Sensing Cognitive Radios. Once a bandwidth is available, if the available bandwidth is wide enough we have two options:

- To transmit bulk data at higher data rate e.g. real time applications like Mobile Services.

- To transmit small packets of data with high accuracy, required in Emergency Services (time bound emergency information should take care of data reduction to minimum possible size so as to utilize low data rates where BER is low).

Present work focuses on the second option for improving the BER performance of the system[3].

The first step in modelling was to prepare a simple Digital Communication System model to test the encoder decoder design. This was done using Matlab Simulink. After testing the functionality of the codec in the DCOM System a gate level model of the encoder was prepared, noting the fact that Matlab Simulink to HDL Converter supports only the behavioral level blocks. These steps were carried out to test the hardware implementation possibility of the codec.

The Cognitive Cycle begins with Radio Scene Analysis, Scanning for Spectrum Holes. Once the bandwidth is available, in the available bandwidth the transmit frequency is decided. So an MFile for bandwidth selection was written [7], [8]. Now the input signal in a Digital Communication System is a digital data (binary bits), obtained from an analogue source. Common example of analogue source is a Speech signal, which varies from $300 \mathrm{~Hz}$ to $3300 \mathrm{~Hz}$. This will decide how many digital data bits are present over the observation time. In the model data bits are randomly generated to represent random speech signal. The bit generation frequency also changes, considering the variations in human speech. We are not concerned with the amplitude variations. Adaption is with regards to frequency \& it should be shown that it is dependant on channel bandwidth and is independent on the frequencies in Input data. It is therefore that the model as been worked out for varying frequencies of input data \& performs equally well over the entire specified range of frequencies of input data. Thus, the model now takes the shape with three parameters as user defined viz.

1. Input data start frequency,

2. Input data End Frequency and

3. Observation Time.

A simple Digital Communication System for commercial communication working over the available bandwidth was 
modeled with rate $1 / 2$ convolution encoder [5], [6]. This was termed as a non adaptive system, wherein it was found that as Bandwidth increases the BER also increases (graph plotted is shown in fig. 9).

To make the system adaptive i.e. as bandwidth increases one should be able to send data with higher accuracy, the codec algorithm is made efficient with additional bits and additional sequential \& combinational logic. This definitely increases the computational time but drastically reduces the BER. High speed processors should take care of this increase in computational time. Typical values of computation time required for varying number of bits per frame is quoted in Table 1.

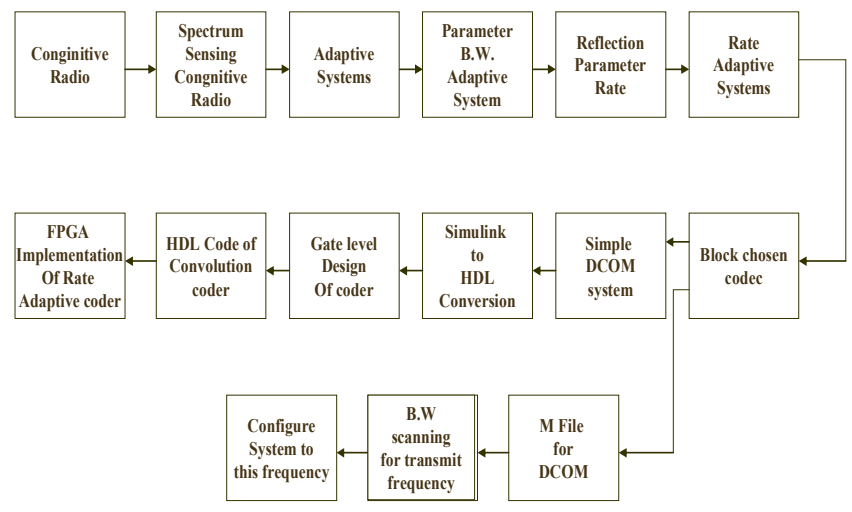

Figure 6. Workflow

TABLE I

Bits Per Frame Vs Computational Time \& CORRESPONDing BER

\begin{tabular}{|l|l|l|l|}
\hline $\begin{array}{l}\text { Sr. } \\
\text { No }\end{array}$ & Bits per Frame & $\begin{array}{l}\text { Computation } \\
\text { Time }\end{array}$ & BER \\
\hline 1 & 5 & $3 \mathrm{~min}$ & $0.1340 \mathrm{E}-2$ \\
\hline 2 & 7 & $7 \mathrm{~min}$ & $0.1184 \mathrm{E}-2$ \\
\hline 3 & 10 & $15 \mathrm{~min} 10 \mathrm{sec}$ & $1.1071 \mathrm{E}-2$ \\
\hline 4 & 15 & 1 hour $36 \mathrm{~min}$ & $0.0750 \mathrm{E}-2$ \\
\hline
\end{tabular}

The reduction in BER with adaptive coder is plotted and the corresponding graph is shown in fig. 10. The convolution encoder in Matlab was HDL coded and the code was downloaded in FPGA [9], [10]. The entire work flow can be briefly depicted in the block diagram shown in fig. 6 .

\section{RESULTS}

The simulation results for the mobile communication system model are as shown in fig. 7. It is plot of BER Vs. Signal to Noise Ratio wherein Decoder iterations is set as a parameter. BER reduces with increase in decoder iterations.

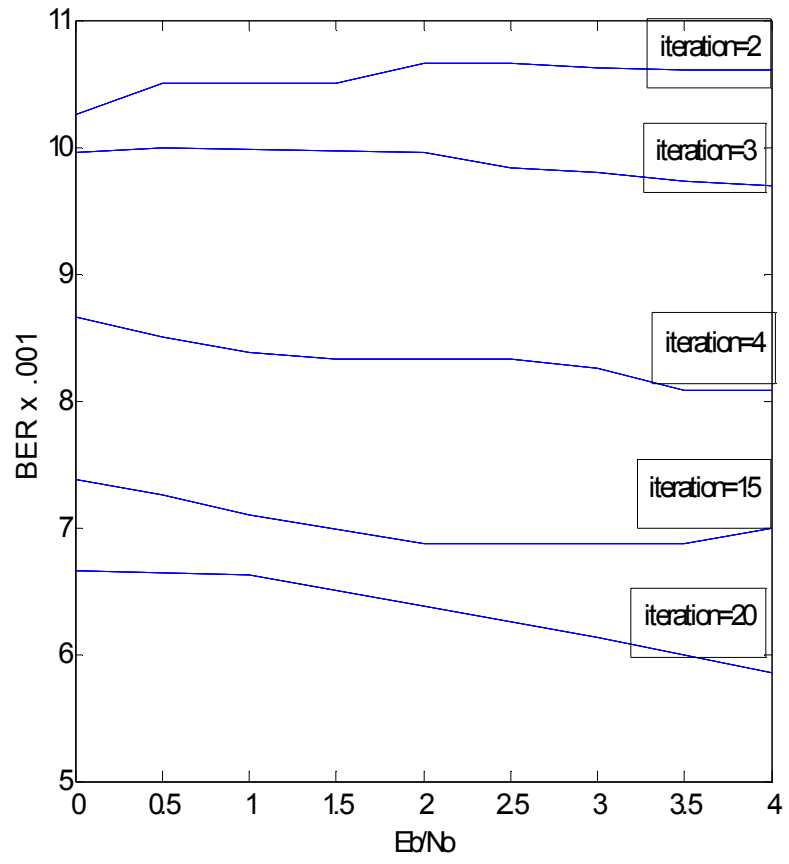

Figure 7. BER Vs. Eb/No, for Mobile Communication system

The Simulation models for WiMan were prepared for following four systems:

1. WiMax using RS+ Convolutional Codec and OQPSK

2. Existing WiMax system(RS + Convolutional) and QPSK

3. WiMax using RS+ Turbo Codec and QPSK

4. WiMax using Turbo Codec and QPSK

Each of them was observed for bit error rate at different values of $\mathrm{S} / \mathrm{N}$ (signal to noise ratio). Graphs of $\mathrm{S} / \mathrm{N}$ versus FER (Frame error rate) were plotted and are as shown in fig 8. 

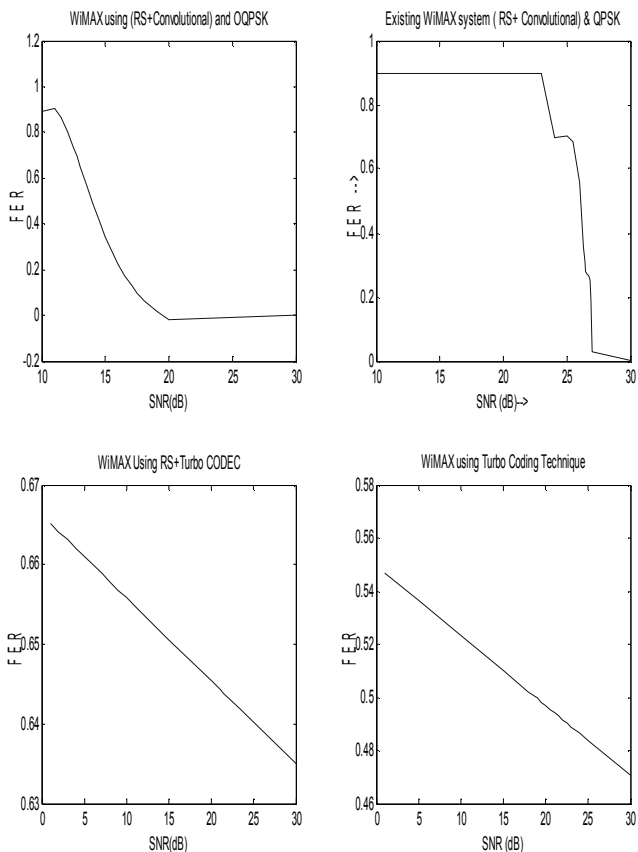

Figure 8. Plots of S/N vs. Frame Error Rate (FER) for different model of WiMax System

From the WiMax Simulation results following Conclusions can be drawn

1. As seen from fig 8 the turbo concatenation with RS gives desired performance of decreasing the bit error rate with increasing $\mathrm{S} / \mathrm{N}$.

2. Using only Turbo codes for the Codec design, works perfect as far as simulation is concerned. But real time systems will introduce Burst errors. To reduce burst errors RS coding is a must.

3. More over, it was observed that every system requires different signal power for the receiver to interpret the data. The Turbo concatenated system works fine with low power signals as compared to only RS codec system.

4. Also the OQPSK system can respond to much low level signals in comparison with the existing QPSK systems and produce a much lower BER.

\section{Results of Cognitive System}

To observe the results in the form of bits on the computer screen and to plot the graphs the working frequencies were scaled down. The model works equally well at high frequencies and was tested for few sample data at high frequencies. The problem faced was that the simulation time taken increases to hours, due to the computational time required in finding the checksum bits that are derived from input bit streams. High frequency is high data bit rate i.e. more no of bits in a given frame. Every checksum bit is derived with a combinational and sequential logic utilizing the input as the input bit stream.

The first model was of a simple non adaptive digital communication system wherein the codec does not adapt to the available bandwidth. It was found that as the bandwidth increases the BER increases, as is clear from graph in fig 9.

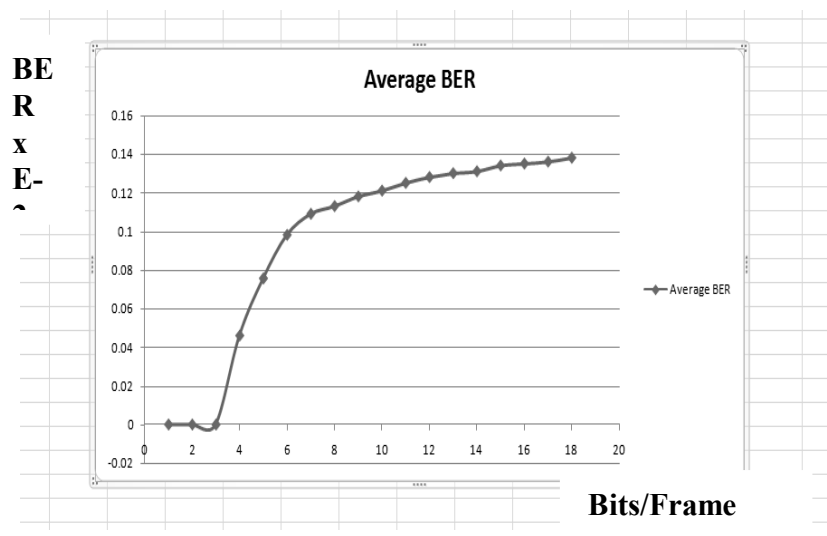

Figure 9. BER on Y-Axis vs. Bits per frame on X-Axis for non adaptive Codec

The second model was prepared with an algorithm to adapt to the increasing bandwidth and utilizing this bandwidth to make the data more secure i.e. to reduce the BER. The graph in fig. 10 shows that the adaptive codec reduces the BER with increase in frame size that has resulted from increase in bandwidth.

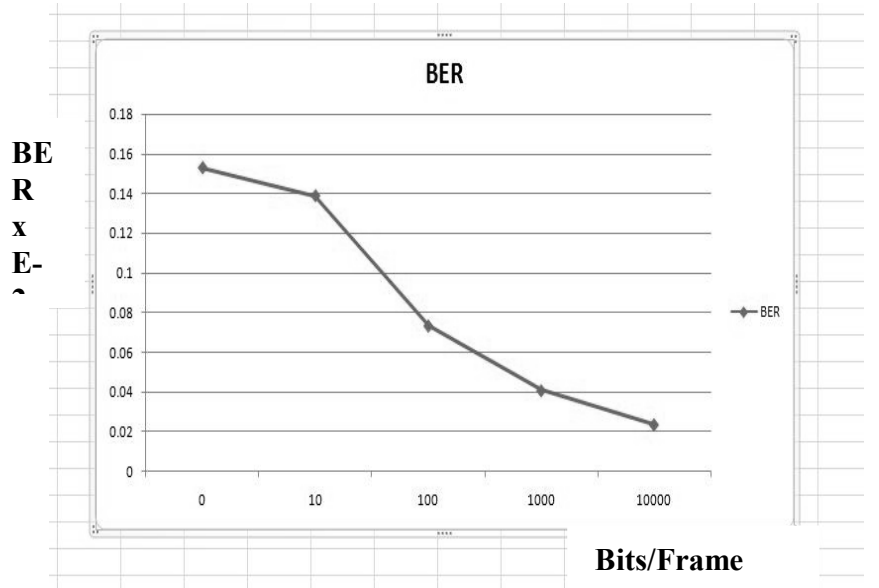

Figure 10. BER on Y-Axis Vs. Bits per frame on X-Axis for adaptive Codec

\section{CONCLUSION}

- For performance optimization one should first think of the parameter to be optimized. Look into the related parameters which improve along with the chosen parameter.

- The most important of all the contradicting ends should be taken care of, their value should remain within the tolerable limits. Application specific conclusions are provided in Section 5. 
- Cognitive radio is an innovative technology proposed to increase spectrum usage by allowing dynamic allocation of the unused spectrum in changing environments. Cognitive users monitor the spectrum and are allowed to use it as long as it does not interfere with primary users to whom it has been licensed. We have proposed the design of a Smart Codec using convolution codes wherein the codec senses the spectrum available and accordingly modifies the algorithm for coding decoding. An increase in bandwidth is utilized for reducing the BER. The hardware encoder had been realized by downloading the HDL code into FPGA.

- $\quad$ The BER performance improvement has been attempted by working over channel coding. One can also work over source coding and use advance Source Coding Techniques like Sub band Coding, Transform Coding, Linear Predictive Coding and Code excited Linear Predictive coding for efficient data compression and send data over smaller BW.

\section{REFERENCES}

[1] C. Berrou, A. Glavieux, and P.Thitmajshima, "Near Shannon limit error-correcting coding and decoding:turbo codes". Proc. IEEE Int Conf. Communication, Geneva, Switzerland, (May 1993)

[2] R. D. Raut and Dr. K. D. Kulat, . "Appication Based Criterion For Selection of Error Control Code". International Conference On Emerging Technologies in Telecommunication Convergence(ICETT-07, Jan 10-12, 2007).

[3] R. D. Raut and Dr. K. D. Kulat, "Spectrum Sensing Smart Codec Design for Cognitive Radio:, The $8^{\text {th }}$ WSEAS International Conference on System Simulation in Engineering", Genova, Italy, Oct 17 th- $19^{\text {th }}$ (paper Selected \& nominated in the best $30 \%$ category for publication in WSEAS Journal).
[4] Jan F. Akyildiz \& etal, “ A Survey on Spectrum Management in Cognitive Radio Netwarks", IEEE Communication Magazine, vol 46, pp. 40-48, April 2008.

[5] R.D. Raut and K. D.Kulat, "Optimal Coec Design for Mobile Communication", TECHNIA, International Journal of Computing Science \& communication Technologies, vol 1, no. 1, pp.. 20-24, July 2008.

[6] R. D. Raut and K. D. Kulat, " Novel approach: Codec design for WiMax system”, IEEE Explore, 18-20 Dec,2008Tavel, P. 2007 Modeling and Simulation Design. AK Peters Ltd.

[7] Matlab 7 Getting Started Guide, Available: http://www.Mathwaorks.com

[8] Simulink Fixed Point 5 User's Guide, Available: http://www.Mathwaorks.com

[9] Matlab 7 Getting Started Guide, Available: http://www.Mathwaorks.com

[10] Simulink Fixed Point 5 User's Guide, Available: http://www.Mathwaorks.com

[11] Tomoaki Ohtsuki, "Rate Adaptive Indoor Infrared Wireless Communication Systems Using Repeated and Punctured Convolutional Codes", IEEE, 1999.Forman, G. 2003. An extensive empirical study of feature selection metrics for text classification. J. Mach. Learn. Res. 3 (Mar. 2003), 12891305.

[12] Marco Chiani, " Coexistence of Ultra- Wide band and other Wireless Systems: the Path Towards Cognitive Radio", UWB Workshop, Ferrara: 2008.

[13] Tomoaki Ohtsuki, "Rate Adaptive Indoor Infrared Wireless Communication Systems Using Repeated and Punctured Convolutional Codes", IEEE, 1999. 